\title{
ORTHOPADDIC SURGERY
}

\section{Radial Aplasia Syndrome - A Case Report}

\author{
R. D. Wammanda, W. N. Ogala and *A. I. Mamman \\ Departments of Paediatrics and *Haematology, Ahmadu Bello University \\ Teaching Hospital, \\ Zaria.
}

\begin{abstract}
A four day-old girl presented with abnormality of both upper limbs. Pregnancy and delivery had been uneventful. Clinical, radiological and hạematological evaluations were consistent with radial aplasia syndrome. The case is reported as a rare congenital malformation of unknown aetiology (Nig J Surg Res 2000; 2:92-94)
\end{abstract}

KEY WORDS: Radial aplasia syndrome

\section{Introduction}

Congenital absence of the radius/radii may be associated with many other congenital anomalies including haematological, genitourinary and other skeletal anomalies. When the radial aplasia is associated with pancytopaenia and multiple anomalies, the condition is termed Fanconi anaemia. When it is associated with congenital hypoplastic thrombocytopacnia with other cell lines being normal, it is termed thrombocytopaenia absent radii (TAR) syndrome. This is an autosomal recessive disorder characterised by a haemorrhagic diathesis, which usually appears within the first few days of life although occasionally not until the fourth weck of life. ${ }^{1,2}$

Radial aplasia without haematological involvement is designated as radial aplasia syndrome $e^{3.4}$ or radial club hand. ${ }^{2.5}$ This is an uncommon condition, which has not been previously widely reported. We report one such case.

Case Report

A four-day old girl was brought to the paediatric out patient department by her mother because of an abnormality observed in both hands at birth. The baby was delivered at term following an uneventful pregnancy to a twenty-five-year old woman. Her mother had received antenatal care at a private

Reprint requests to:, Dr. R. D. Wammanda Department of Paediatrics, A. B. U. Teaching Teaching Hospital, Zaria. 
hospital where she was also delivered. There was no history of maternal illness during the pregnancy. Throughout the pregnancy, the mother received only routine haematinics (folic acid and iron preparations) and antimalarial drugs (pyrimethamine) in normal prophylactic doses. Delivery was uneventful. The baby passed meconium and urine within the first 24 hours of life. She is the second child of a monogamous family with no history of consanguinity. The first child who was 2 years old was normal. There was no history of similar anomaly in both families.

On examination, the patient was a normal looking neonate except for the obvious bilateral anomaly in both upper limbs. She was alert, pink and active. There were no rashes. She weighed $3.2 \mathrm{~kg}$ with a length of $54 \mathrm{~cm}$ and occipito-frontal circumference of $33 \mathrm{~cm}$. Anterior fontanelle measured $3 \mathrm{~cm} \mathrm{x}$ $4 \mathrm{~cm}$ but the sagittal suture was already closed.

There was bilateral shortening of both forearms with fixed flexion deformity at the elbow joints. Both hands were deviated radially with hypermobility of the wrist joints. The thumbs and thenar eminences were present and movement in the hands were normal. There was no clinical evidence of congenital heart disease and kidneys were not palpably enlarged. The other systems were normal.

Radiography of the forearm showed bilateral absence of the radii (Figure 1). The ulna bones were bowed and their distal ends inclined to the radial side. The metacarpals and phalanges were normal. A skeletal survey revealed no other abnormalities in the baby. The haematocrit was $36 \%$ while the total white cell and platelet counts were $7,000 / \mathrm{mm}^{3}$ and $179,00 / \mathrm{mm}^{3}$ respectively. These were repeated on two other occasions and found to be within normal limits. Bone marrow aspiration biopsy. performed on the 43rd day of life showed a normal marrow with adequate megakaryocytic cells. It was not possible to carry out chromosomal studies at the time. The baby remained healthy until she was lost to follow up at the neonatology clinic.

\section{Figure 1: Bilateral absence of the radii}

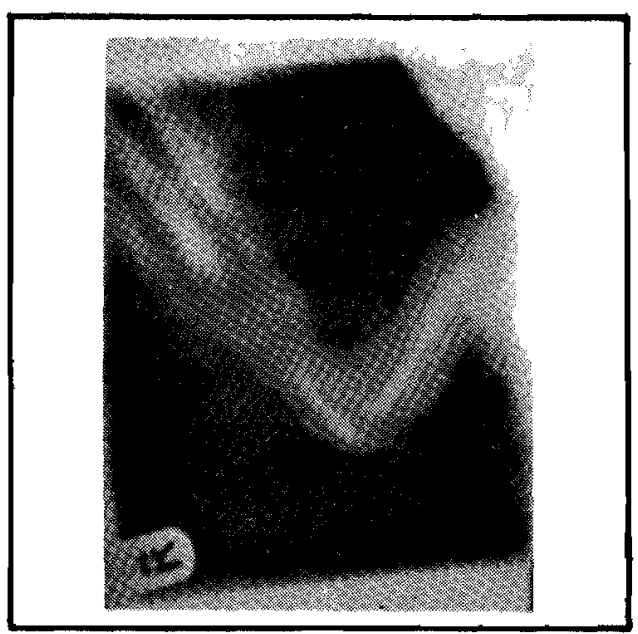

\section{Discussion}

In radial aplasia syndrome, there is a wide variation of deformities and disabilities affecting both arms in about one half of the affected patients. ${ }^{4}$ In a classical case like the one reported, the radius is either absent or severely shortened, the ulna being short and bowed so that its distal end is inclined to the radial side. The wrist is unstable and the hand lies in fixed radial deviation uith palmer flexion. The elbow is stable but frequently lacks extension or flexion to a variable degree. No haematological abnormalities are 
described. Our patient fits well into the syndrome.

The occasional associated abnormalities described in this syndrome are the absence of the thumb or its presence in a rudimentary form and fixed flexion deformity of the proximal interphalangeal joints with variable finger power. Although this condition has been associated with thalidomide poisoning, it has been reported before and after the drug was withdrawn from circulation. ${ }^{4}$ There was no history suggestive of thalidomide poisoning in our patient.

Various modes of treatment have been tried. ${ }^{2,}+$ These include initial stretching and splinting of the hand towards ulna deviation with dorsiflexion. This is followed by radial release and ulna osteotomy, which allows the hand to be set in line with the forearm. Centralising the carpus over the end of the ulna after removal of a variable number of the carpal bones without interference with the growth plate has also been attempted with some success. ${ }^{2.4}$ This procedure stabilises the wrist, retains movement and does not inhibit growth. However, later development of arthrodesis is a common complication. ${ }^{4}$ In some untreated cases, these patients may undergo natural functional adaptatation to make a remarkable good use of the deformed limb., 5

There are few reports of radial aplasia in Nigeria. Adeyokunu ${ }^{6}$ reported five patients with radial aplasia among Nigerian children during a ten-year study period. In all these patients, there were associated amegakoryocytic thrombocytopaenia and were therefore TAR syndrome cases. This case is reported therefore to increase our awareness in this rare congenital malformation.

\section{References}

1. Lecler JC, Toth J. Thrombocytopaenia with absent radii. Can Med Assoc $J$ 1982; 126: 506-508.

2. Thompson GH, Scoles PV. Radial clubhand. In: Behrman RE, Kliegman RM, Arvin AM (eds). Nelson's textbook of paediatrics. Saunders, Philadelphia, 1996; 1952.

3. Sohi BK, Mukherjee, Ghosh M.K. Radial aplasia syndrome. Indian Paediatr 1979; 16: 463 - 464.

4. Roberts L. Radial aplasia syndromes, In: Orthopaedics in infancy and childhood. Butterworts, London, 1971; 163-166.

5. Sharraord WJW. Paraxial radial hemimelia. In: Paediatric orthopaedics and fractures. Blackwell Scientific Publications, London, 1971;107-110.

6. Adeyokunu A. Radial aplasia and amegakaryocytic thrombocytopenia (TAR Syndrome) among Nigerian children. Am J Dis Child 1984; 138: 346-348. 